\section{Fatores associados ao atraso do desenvolvimento motor de crianças prematuras internadas em unidade de neonatologia}

\section{Factors associated with late motor development in premature children admitted to a neonatology unit}

Alessandra Teixeira da Câmara Araújo 1 Sophie Helena Eickmann 2 Sônia Bechara Coutinho 3

\begin{abstract}
Objectives: to assess the frequency of late motor development in premature children admitted to a neonatal unit and to identify associated factors.

Methods: a descriptive analytical study carried out at the Neonatal Unit of a high-risk maternity hospital in Recife, Brazil. Motor development was assessed using the Test of Infant Motor Performance, in 98 preterm children with a minimal post-conception age of 34 weeks, during admission to hospital, between January and June 2009. Associations were examined between variables relating to the mother, the newborn, the care provided and motor development.

Results: the mean post-conception age on evaluation was 37 weeks and $39.8 \%$ of the children had some kind of impaired motor development (atypical or suspected to be atypical). Factors significantly associated with changes in motor development were: lower birth weight; higher age on evaluation; greater number of prenatal sessions; occurrence of hypoxia and bronco-pulmonary dysplasia; longer stay in ICU and neonatal unit. There was a significant trend for motor development to worsen with prolonged use of oxygen-therapy and mechanical ventilation.

Conclusions: premature children admitted to hospital may show early signs of delayed motor development of a severe or borderline nature. Birth weight, neonatal morbidities and care received at the neonatal unit were the factors that most strongly influenced the outcome. These results justify initiation of preventive strategies and stimulation while the child is still in hospital.
\end{abstract}

Key words Infanti, premature, Child development, Developmental disabilities, Intensive care units neonatal, Early diagnosis
1 Departamento de Reabilitação e Desporto. Universidade Salgado de Oliveira. Av. Mascarenhas de Moraes, 2169, Imbiribeira. Recife, PE, Brasil. CEP: 51.170-000. E-mail: alessandracamara@ig.com.br 2,3 Departamento Materno-Infantil. Universidade Federal de Pernambuco. Recife, PE, Brasil.

\section{Resumo}

Objetivos: verificar a frequência do atraso do desenvolvimento motor em crianças prematuras internadas em unidade neonatal e identificar os fatores associados

Métodos: estudo descritivo e analítico, realizado na Unidade Neonatal de uma maternidade de alto risco em Recife, Brasil. Avaliou-se o desenvolvimento motor, através do Test of Infant Motor Performance, de 98 crianças nascidas pré-termo com idade pósconceptual mínima de 34 semanas, durante a internação, entre janeiro e julho de 2009. Analisaram-se associações entre variáveis maternas, neonatais e assistenciais com desenvolvimento motor.

Resultados: a média de idade pós-conceptual na avaliação foi de 37 semanas e 39,8\% das crianças apresentaram desenvolvimento motor alterado (atipico ou suspeito). Fatores significativamente associados ao desenvolvimento motor alterado foram: menor peso ao nascer; maior idade na avaliação; maior número de consultas pré-natais; ocorrência de hipóxia e displasia broncopulmonar; maior tempo de permanência na unidade de terapia intensiva e unidade neonatal. Houve tendência significante a um pior desenvolvimento motor com uso de oxigenoterapia e ventilação mecânica prolongadas.

Conclusões: crianças prematuras internadas podem apresentar atraso precoce do desenvolvimento motor, de forma grave ou limitrofe. Peso ao nascer, morbidades neonatais e assistência recebida na unidade neonatal foram os fatores que mais influenciaram o desfecho. Esses resultados justificam o início de estratégias preventivas e estimulação ainda no período de internação.

Palavras-chave Prematuro, Desenvolvimento infantil, Deficiências do desenvolvimento, Unidades de Terapia Intensiva Neonatal, Diagnóstico precoce 


\section{Introdução}

A prematuridade é reconhecida como um importante fator de risco para distúrbios do desenvolvimento motor, 1,2 uma vez que promove uma interrupção na progressão do desenvolvimento das estruturas cerebrais, podendo afetar eventos importantes, como a sinaptogênese e a mielinização. ${ }^{3}$ Ademais, a presença de comorbidades comuns na prematuridade (como hipóxia e displasia broncopulmonar) e o uso de alguns métodos assistenciais por períodos prolongados, tais como oxigenoterapia e ventilação mecânica invasiva, podem favorecer lesões neurológicas, provocando alterações transitórias ou duradouras, as quais podem variar desde um discreto atraso na aquisição das etapas motoras até o desenvolvimento de paralisia cerebral.1,4-6

Para muitos recém-nascidos prematuros, a permanência em Unidade de Terapia Intensiva (UTI) é essencial à manutenção da vida. Porém, no ambiente da unidade neonatal, a exposição constante à estimulação excessiva (ruídos, luminosidade, procedimentos dolorosos, entre outros) em crianças neurofisiologicamente imaturas pode desencadear alterações motoras e hemodinâmicas. 7,8

Se por um lado as influências negativas desse meio externo conferem maior vulnerabilidade ao cérebro do prematuro num período crítico para o desenvolvimento do seu sistema nervoso, tornandoo mais susceptível aos problemas do desenvolvimento, por outro lado a intensa neuroplasticidade dos primeiros meses favorece uma melhor resposta à estimulação. 9 Dessa forma, o diagnóstico precoce dos distúrbios do desenvolvimento torna-se fundamental, pois possibilita a antecipação das ações de intervenção, podendo reduzir os riscos de sequelas. ${ }^{9,10}$ Entretanto, em recém-nascidos prematuros internados em unidade neonatal, a avaliação apresenta-se como um desafio, não só devido às rápidas modificações do desenvolvimento mas também às influências das intervenções terapêuticas no comportamento do bebê. Portanto, é importante a utilização de instrumentos que tenham sensibilidade para identificar pequenas variações e, ainda, que possam ser aplicados em idades precoces. ${ }^{11}$ Assim, o objetivo do presente estudo foi verificar a frequência do atraso do desenvolvimento motor numa população de crianças prematuras internadas em unidade neonatal e identificar os fatores maternos, neonatais e assistenciais associados a esse atraso.

\section{Métodos}

Realizou-se estudo descritivo, com componente analítico, em uma maternidade pública de referência no atendimento às gestantes de risco, em Recife, Brasil. A população estudada foi composta por 98 crianças com idade pós-conceptual a partir de 34 semanas, internadas na Unidade Neonatal e avaliadas antes da alta hospitalar, no período de internação entre janeiro e julho de 2009. O tamanho da amostra foi determinado pelo período de observação e as crianças foram selecionadas para a pesquisa à medida que preenchiam os seguintes critérios de inclusão: idade gestacional inferior a 37 semanas e, no momento da avaliação, idade pós-conceptual mínima de 34 semanas; estabilidade clínica e hemodinâmica, caracterizada por frequência respiratória entre 30-60 rpm (respirações por minuto), frequência cardíaca entre 120-160 bpm (batimentos por minuto), nível de saturação de oxigênio acima $89 \%$, ausência de sinais de desconforto respiratório (Boletim de Silverman Anderson $<4$ ); ausência de cianose, palidez e de sinais de dor e/ou desconforto; tempo mínimo de internação na unidade neonatal de três dias.

Excluíram-se crianças com síndromes genéticas (diagnosticadas ou sob suspeita), distúrbios neurológicos, distúrbios auditivos ou visuais (diagnosticados através da triagem auditiva neonatal e da oftalmoscopia indireta, exames realizados rotineiramente no serviço), malformações graves e as que estavam em assistência ventilatória mecânica e/ou com acesso venoso no momento da avaliação.

As informações maternas e os dados relativos às crianças foram coletados nos prontuários dos neonatos e registrados em formulários de pesquisa padronizados e pré-codificados, contendo o histórico gestacional (número de consultas durante o pré-natal e morbidades maternas), características clínicas e antropométricas do recém-nascido (sexo, idade gestacional, peso ao nascer, Apgar aos 5 minutos, perímetro cefálico, adequação peso/idade gestacional), além do histórico de morbidades, assistência e evolução no período de internação.

A idade pós-conceptual no momento da avaliação foi calculada através da fórmula: idade pósconceptual (semanas) $=$ idade gestacional (semanas, determinada pela equipe médica com base no último dia da menstruação (DUM), ultrassonografia ou, ainda, através dos métodos New Ballard ou Capurro) + idade cronológica (tempo de vida, em semanas).

As exposições pré-natais estudadas foram: infecção (infecção do trato urinário e corioamnionite), diabetes gestacional, hemorragia vaginal e 
hipertensão maternas. Quanto às exposições pósnatais, foram consideradas: hipóxia/asfixia perinatal, hiperbilirrubinemia e infecção/sepse neonatal; hemorragia intracraniana (confirmada através da ultrassonografia transfontanela); displasia broncopulmonar (diagnosticada como a necessidade de oxigênio suplementar acima de $21 \%$ por um período maior ou igual aos 28 dias de vida12); tempos de hospitalização na unidade neonatal e de permanência na unidade de terapia intensiva (registrados em dias); uso de surfactante pulmonar; tempo de utilização de oxigenoterapia e de ventilação mecânica invasiva (em dias). Os diagnósticos das exposições pré e pós-natais, coletados a partir dos prontuários dos recém-nascidos, foram efetuados pela equipe médica responsável, conforme critérios especificados nas pautas de rotinas médicas do serviço.

A variável de desfecho foi o diagnóstico de desenvolvimento motor avaliado através do Test of Infant Motor Performance (TIMP). Com o intuito de minimizar viés de avaliação, uma única pesquisadora, previamente treinada, examinou todas as crianças do estudo. Cada avaliação durou, em média, 30 minutos e foi realizada durante o período diurno, com a criança alerta, respeitando o intervalo mínimo de uma hora após a dieta.

O TIMP foi escolhido por ser um instrumento adequado para a avaliação de recém-nascidos prematuros e por poder ser aplicado em crianças internadas, 13 desde que estáveis, tendo demonstrado ser um instrumento capaz de identificar crianças em risco para atraso do desenvolvimento motor a longo prazo. $14 \mathrm{O}$ teste demonstra sensibilidade às mudanças rápidas de comportamento do bebê, 11 pois permite a identificação de novas aquisições motoras a cada duas semanas de vida, possibilitando a avaliação e comparação do desenvolvimento da criança com grupos normativos com pequena diferença de idade. ${ }^{13} \mathrm{O}$ TIMP consiste de 42 itens, divididos em duas subescalas: a) Escala de Observação: avalia a intenção da criança em modificar posições, orientar a cabeça e tronco e iniciar movimentos de segmentos corporais; b) Escala de Resposta: avalia a coordenação da resposta postural da criança diante de diferentes situações em variadas posições. Os 13 itens da Escala de Observação são registrados através de observação direta da criança, num período de 1 a 3 minutos, durante sua movimentação espontânea e com mínimo manuseio. Cada item observado (presente) recebeu o escore 1 e os itens não observados (ausentes) receberam o escore 0. A Escala de Resposta é composta por 29 itens com até seis níveis hierárquicos de dificuldade e a criança recebeu, em cada item, o escore correspondente ao nível atingido no momento da avaliação (0-6).

Os valores obtidos nas duas subescalas foram somados para compor o escore total, o qual foi comparado à tabela de padrões de desempenho motor presente no manual do teste. $\mathrm{O}$ escore $\mathrm{z}$ foi, então, calculado, tomando-se como base o escore total e o desvio-padrão esperado para cada idade corrigida. ${ }^{13}$ Consideraram-se como casos de desenvolvimento atípico as crianças cujo escore $\mathrm{z}$ foi menor que -1 desvio padrão e como desenvolvimento motor típico aquelas com escore $\mathrm{z}$ maior ou igual a $-0,5$ desvio-padrão. Os neonatos que apresentaram escore $\mathrm{z}$ entre $-0,5 \mathrm{e}-1$ desvio-padrão foram diagnosticados como suspeitos de atraso de desenvolvimento motor.

Para fins de análise estatística, as crianças diagnosticadas como suspeitas de atraso foram agrupadas àquelas com diagnóstico de desenvolvimento motor atípico. Para esse grupo, foi criada uma nova categoria, designada como desenvolvimento motor alterado cujos resultados foram comparados com os do grupo de desenvolvimento motor típico.

O processamento dos dados realizou-se através de dupla entrada para avaliação da consistência da digitação utilizando-se o software Epi-Info 6.04. Este software também foi utilizado para a análise estatística. O teste do qui-quadrado de Pearson foi empregado para avaliar as associações entre as variáveis maternas, neonatais e assistenciais com a variável de desfecho. Nos casos em que a frequência esperada foi menor que cinco em mais de $20 \%$ das caselas da tabela de contingência, utilizou-se o teste Exato de Fisher. O teste do qui-quadrado para tendência foi utilizado para confirmar a significância estatística na tendência linear de associação entre as variáveis categóricas. Em todas as análises adotouse o nível de significância de 5\%.

As mães das crianças elegíveis consentiram, previamente, com a sua participação na pesquisa, assinando o Termo de Consentimento Livre e Esclarecido, conforme resolução $n^{\circ} 196 / 96$ do Conselho Nacional de Saúde. O estudo foi aprovado pelo Comitê de Ética em Pesquisa do Centro Integrado de Saúde Amaury de Medeiros/CISAM (parecer 073/08). As genitoras cujos filhos apresentaram desenvolvimento alterado receberam orientações para a realização de estimulação motora.

\section{Resultados}

Durante o período pesquisado, estiveram internadas na unidade neonatal 312 crianças. Dessas, 136 preencheram os critérios de inclusão e 104 foram 
excluídas. Com as perdas relacionadas aos óbitos (67), às transferências (05) e às altas anteriores à realização da avaliação (38), a amostra totalizou 98 crianças (correspondendo a $72 \%$ da população internada no período e que preenchia os critérios de inclusão). Das crianças avaliadas, 39,8\% (39/98) apresentaram desenvolvimento motor alterado segundo o Test of Infant Motor Performance, considerando-se como alterados os resultados suspeitos conjuntamente com os atípicos. A Tabela 1 apresenta os resultados do teste, considerando as três categorias de desenvolvimento motor.

Com relação às características maternas, a idade mínima das genitoras foi de 14 e a máxima de 43 anos. A maioria das mães realizou o pré-natal e apenas três não receberam acompanhamento médico durante a gravidez. As complicações gestacionais mais frequentes foram as infecções $(53,7 \%)$, seguidas por hipertensão arterial $(23,2 \%)$ e hemorragia vaginal $(18,8 \%)$. Apenas quatro mães $(4,2 \%)$ apresentaram diabetes gestacional. Quanto aos neonatos, a idade gestacional mínima foi de 25 semanas e o peso ao nascer variou de $740 \mathrm{~g}$ a $2680 \mathrm{~g}$. Um percentual de $74,5 \%$ da amostra era de recémnascidos pequenos para a idade gestacional. As crianças foram avaliadas entre 34 e 43 semanas de idade pós-conceptual, com média de 37 semanas. A Tabela 2 caracteriza a amostra quanto ao perfil das crianças e das mães.

A idade da mãe e as morbidades maternas ocorridas no período gestacional não estiveram significativamente associadas ao desfecho. Entretanto, conforme apresentado na Tabela 3 , houve uma relação inversa entre a maior frequência de consultas no pré-natal e piores resultados na avaliação do desenvolvimento motor.

Tabela 1

Avaliação do desenvolvimento motor pelo Test of Infant Motor Performance (TIMP) em crianças prematuras internadas em unidade de neonatologia.

\begin{tabular}{lcccccc}
\hline Desenvolvimento Motor & $\mathbf{N}$ & $\%$ & Escore z mínimo & Escore z máximo & Médias (escore z) & DP \\
\hline Típico & 59 & 60,2 & $-0,46$ & 1,25 & 0.06 & 0,38 \\
Suspeito & 26 & 26,5 & $-1,00$ & $-0,54$ & $-1,06$ & -1.14 \\
Atípico & 13 & 13,3 & $-1,43$ & & 0,13 \\
\end{tabular}

Tabela 2

Características relacionadas às mães, complicações gestacionais e características biológicas das crianças prematuras internadas em unidade de neonatologia.

\begin{tabular}{lcc}
\hline Características maternas e da criança & $\overline{\mathbf{X}} \pm \mathbf{D P}$ & Total \\
\hline Idade da mãe (anos) & $24,5 \pm 7,3$ & 98 \\
$\mathrm{~N}^{\circ}$ de consultas pré-natal* & $4,7 \pm 2,0$ & 90 \\
Peso ao nascer (g) & $1627 \pm 419$ & 98 \\
Idade gestacional (semanas) & $34,2 \pm 2,0$ & 98 \\
Perímetro cefálico (cm) ${ }^{\dagger}$ & $29,3 \pm 2,5$ & 86 \\
Apgar 5’ & $8,8 \pm 1,1$ & 97 \\
Idade pós-conceptual (semanas)§ & $37,2 \pm 1,8$ & 98
\end{tabular}

*8 prontuários sem informação; †12 prontuários sem informação; ¥1 prontuário sem informação; §idade corrigida no momento da avaliação. 
Desenvolvimento motor de crianças prematuras internadas em unidade de neonatologia segundo as características maternas e biológicas da criança.

\begin{tabular}{|c|c|c|c|c|c|c|c|}
\hline \multirow{3}{*}{ Variáveis } & \multirow{2}{*}{\multicolumn{2}{|c|}{$\begin{array}{c}\text { Total } \\
(\mathrm{N}=98)\end{array}$}} & \multicolumn{4}{|c|}{ Resultado do TIMP } & \multirow{3}{*}{$p$} \\
\hline & & & \multicolumn{2}{|c|}{ Típico $(\mathrm{N}=59)$} & \multicolumn{2}{|c|}{ Alterado $(\mathrm{N}=39)$} & \\
\hline & $\mathrm{n}$ & $\%$ & $\mathrm{n}$ & $\%$ & $\mathrm{n}$ & $\%$ & \\
\hline \multicolumn{8}{|c|}{ Consultas no pré-natal* } \\
\hline $0-3$ & 31 & 34,4 & 25 & 80,6 & 6 & 19,4 & 0,01 \\
\hline $4-6$ & 42 & 46,7 & 20 & 47,6 & 22 & 52,4 & \\
\hline $7-10$ & 17 & 18,9 & 9 & 52,9 & 8 & 47,1 & \\
\hline \multicolumn{8}{|l|}{ Sexo } \\
\hline Masculino & 50 & 51,0 & 29 & 58,0 & 21 & 42,0 & 0,80 \\
\hline Feminino & 48 & 49,0 & 30 & 62,5 & 18 & 37,5 & \\
\hline \multicolumn{8}{|c|}{ Idade gestacional (semanas) } \\
\hline 25 & 21 & 21,4 & 9 & 42,9 & 12 & 57,1 & 0,16 \\
\hline 33 & 20 & 20,4 & 14 & 70,0 & 6 & 30,0 & \\
\hline 35 & 57 & 58,2 & 36 & 63,2 & 21 & 36,8 & \\
\hline \multicolumn{8}{|c|}{ Peso ao nascer (g) } \\
\hline $400-999$ & 7 & 7,2 & 2 & 28,6 & 5 & 71,4 & $0,004 s$ \\
\hline 1000-1499 & 21 & 21,4 & 9 & 42,9 & 12 & 57,1 & \\
\hline 1500-1999 & 55 & 56,1 & 36 & 65,5 & 19 & 34,5 & \\
\hline$\geq 2000$ & 15 & 15,3 & 12 & 80,0 & 3 & 20,0 & \\
\hline \multicolumn{8}{|c|}{ Adequação PN x IG } \\
\hline PIG & 73 & 74,5 & 41 & 56,2 & 32 & 43,8 & 0,25 \\
\hline AIG & 25 & 25,5 & 18 & 72,0 & 7 & 28,0 & \\
\hline \multicolumn{8}{|c|}{ Idade pós-conceptualt (semanas) } \\
\hline $34-36$ & 32 & 32,7 & 25 & 78,1 & 7 & 21,9 & 0,02 \\
\hline$\geq 37$ & 66 & 67,3 & 34 & 51,5 & 32 & 48,5 & \\
\hline
\end{tabular}

TIMP = Test of Infant Motor Performance; PN = peso ao nascer; IG = idade gestacional; PIG = pequeno para a idade gestacional; AIG = adequado para a idade gestacional; *8 prontuários sem informação; †ldade corrigida da criança no momento da avaliação; Steste qui-quadrado de tendência.

Segundo as características dos recém-nascidos, a análise bivariada evidenciou piores resultados de desenvolvimento em crianças com menor peso ao nascer e maior idade pós-conceptual, verificando-se uma tendência para a redução dos percentuais alterados, conforme aumentava o peso ao nascer. Observou-se, ainda, uma proporção maior de crianças com desenvolvimento atípico conforme diminuía a idade gestacional e entre aquelas pequenas para a idade gestacional, porém sem significância estatística (Tabela 3).

Quando analisados os resultados do teste de desenvolvimento em relação à exposição às morbidades neonatais, houve uma associação significante entre ocorrência de hipóxia/asfixia perinatal e displasia broncopulmonar com o desenvolvimento motor alterado. Observou-se, ainda, uma maior proporção de crianças com desenvolvimento alterado tanto entre as que desenvolveram infecção, como entre aquelas que apresentaram algum grau de hemorragia intracraniana, embora esses resultados não tenham sido estatisticamente significantes (Tabela 4).

Quanto aos cuidados oferecidos na unidade neonatal, $21(21,6 \%)$ recém-nascidos fizeram uso de surfactante pulmonar exógeno e $88(89,8 \%)$ necessitaram de oxigenoterapia (média de 8,6 dias). Trinta e seis crianças foram internadas na unidade de terapia intensiva neonatal, com tempo médio de permanência de 23 dias e maior período de 89 dias. Dessas, 24 necessitaram de ventilação mecânica invasiva, sendo que a média de utilização foi de 9 dias, com variação de 1 a 28 dias. O tempo total de hospitalização no momento da avaliação variou de 3 a 97 dias, com média de 21 dias (dados não apresentados em Tabela).

A utilização de oxigenoterapia e ventilação invasiva, bem como o tempo de permanência na unidade de terapia intensiva e na unidade neonatal, apresentaram associação significante com a alteração do desenvolvimento motor. A análise de tendência linear confirmou uma propensão a piores resultados 
Desenvolvimento motor de crianças prematuras internadas em unidade de neonatologia, segundo a evolução clínica e cuidados recebidos na unidade neonatal.

\begin{tabular}{|c|c|c|c|c|c|c|c|}
\hline \multirow{3}{*}{ Morbidades/Evolução } & \multirow{2}{*}{\multicolumn{2}{|c|}{$\begin{array}{l}\text { Total } \\
(\mathrm{N}=98)\end{array}$}} & \multicolumn{4}{|c|}{ Resultado do TIMP } & \multirow{3}{*}{$p$} \\
\hline & & & \multicolumn{2}{|c|}{ Típico $(\mathrm{N}=59)$} & \multicolumn{2}{|c|}{ Alterado $(\mathrm{N}=39)$} & \\
\hline & $\mathrm{n}$ & $\%$ & $\mathrm{n}$ & $\%$ & $\mathrm{n}$ & $\%$ & \\
\hline \multicolumn{8}{|l|}{ Hipóxia } \\
\hline Moderada ou grave & 6 & 6,1 & 1 & 16,7 & 5 & 83,3 & $0,03 \|$ \\
\hline Nenhuma ou leve & 92 & 93,9 & 58 & 63,0 & 34 & 37,0 & \\
\hline \multicolumn{8}{|l|}{ Infecção/Sepse* } \\
\hline $\operatorname{Sim}$ & 49 & 52,1 & 24 & 49,0 & 25 & 51,0 & $0,05 \uparrow$ \\
\hline Não & 47 & 47,9 & 32 & 71,1 & 13 & 28,9 & \\
\hline \multicolumn{8}{|l|}{ Displasia broncopulmonar } \\
\hline Sim & 9 & 9,2 & 2 & 20,2 & 7 & 77,8 & $0,03 \|$ \\
\hline Não & 89 & 90,8 & 57 & 64,0 & 32 & 36,0 & \\
\hline \multicolumn{8}{|l|}{ Hiperbilirrubinemia } \\
\hline $\operatorname{Sim}$ & 94 & 95,9 & 56 & 59,6 & 38 & 40,4 & $1,00 \|$ \\
\hline Não & 4 & 4,1 & 3 & 75,0 & 1 & 25,0 & \\
\hline \multicolumn{8}{|c|}{ Hemorragia intracraniana ${ }^{\dagger}$} \\
\hline Sim & 10 & 15,9 & 4 & 40,0 & 6 & 60,0 & $0,50 \|$ \\
\hline Não & 53 & 84,1 & 29 & 54,7 & 24 & 45,3 & \\
\hline \multicolumn{8}{|l|}{ Uso de surfactante $\ddagger$} \\
\hline Sim & 21 & 21,6 & 10 & 47,6 & 11 & 52,4 & $0,25 \uparrow$ \\
\hline Não & 76 & 78,4 & 49 & 64,5 & 27 & 35,5 & \\
\hline \multicolumn{8}{|c|}{ Tempo de oxigenoterapia (dias) } \\
\hline Não usou $\mathrm{O}_{2}$ & 10 & 10,2 & 7 & 70,0 & 3 & 30,0 & $0,001 * *$ \\
\hline $1-7$ & 63 & 64,3 & 45 & 71,4 & 18 & 28,6 & \\
\hline$\geq 8$ & 25 & 25,5 & 7 & 28,0 & 18 & 72,0 & \\
\hline \multicolumn{8}{|l|}{ Tempo de VMA (dias) } \\
\hline Não foi para VMA & 74 & 75,5 & 49 & 66,2 & 25 & 33,8 & $0,02 * *$ \\
\hline $1-7$ & 14 & 14,3 & 7 & 50,0 & 7 & 50,0 & \\
\hline$\geq 8$ & 10 & 10,2 & 3 & 30,0 & 7 & 70,0 & \\
\hline \multicolumn{8}{|l|}{ Tempo na UTIN (dias) } \\
\hline Não foi para UTIN & 62 & 63,2 & 43 & 69,4 & 19 & 30,6 & $0,004 * *$ \\
\hline $1-7$ & 8 & 8,2 & 06 & 75,0 & 2 & 25,0 & \\
\hline$\geq 8$ & 28 & 28,6 & 10 & 35,7 & 18 & 64,3 & \\
\hline \multicolumn{8}{|c|}{ Tempo de hospitalização§ (dias) } \\
\hline $1-14$ & 47 & 48,0 & 35 & 74,5 & 12 & 25,5 & $<0,001 * *$ \\
\hline $15-30$ & 31 & 31,6 & 18 & 58,1 & 13 & 41,9 & \\
\hline$\geq 31$ & 20 & 20,4 & 6 & 30,0 & 14 & 70,0 & \\
\hline
\end{tabular}

TIMP = Test of Infant Motor Performance; UTIN = unidade de terapia intensiva neonatal; VMA = ventilação mecânica assistida; *2 prontuários com informação ignorada; †35 prontuários sem informação; ¥1 prontuário sem informação; §tempo total de internamento na unidade neonatal, até o momento da avaliação; " teste exato de Fisher; १teste do qui-quadrado; **teste do qui-quadrado para tendência. 
de desenvolvimento conforme aumentavam os tempos de internação. Essa tendência foi igualmente observada para o uso prolongado de oxigenoterapia e de ventilação mecânica (Tabela 4).

\section{Discussão}

Na presente pesquisa, a avaliação através do TIMP no período de internação em unidade neonatal evidenciou uma frequência relativamente baixa de crianças prematuras com atraso importante do desenvolvimento motor $(13,3 \%$ com desenvolvimento atípico). Considerando que a amostra utilizada correspondia a uma população de risco para o desenvolvimento, não só pela prematuridade como também devido às comorbidades apresentadas, esse resultado chama a atenção e poderia sugerir que, talvez, a humanização e os avanços no assistencialismo neonatal possam estar contribuindo positivamente para o desenvolvimento de prematuros, diminuindo os casos graves de atraso. Segundo Vandenberg, ${ }^{8}$ um programa de cuidados individualizados baseado no comportamento da criança e associado à assistência médica avançada cria oportunidade para que a criança alcance suas metas de desenvolvimento.

A frequência de alteração do desenvolvimento motor elevou-se consideravelmente $(39,8 \%)$ quando as crianças com desenvolvimento motor atípico foram agrupadas às diagnosticadas como suspeitas, ou seja, àquelas que apresentaram desempenho motor limítrofe ou ligeiramente abaixo da média. Conforme os autores do TIMP, o ponto de corte de 0,5 (que inclui as crianças suspeitas e atípicas) é recomendado para identificação de crianças que devem ser acompanhadas mais de perto ou encaminhados para intervenção. ${ }^{13}$ Uma vez que este foi o propósito clínico final da presente pesquisa, justifica-se, assim, o agrupamento aqui utilizado.

Em um estudo no qual os resultados do TIMP para crianças prematuras foram comparados com os de crianças nascidas a termo, Guimarães et al. 15 encontraram $26,1 \%$ de prematuros com atraso do desenvolvimento. Os autores utilizaram como ponto de corte um $\mathrm{z}$ escore menor que $-0,5$ para definição de desenvolvimento motor atípico, ou seja, o mesmo ponto de corte que o presente estudo usou para definição de desenvolvimento motor alterado. No entanto, deve-se ressaltar que, no trabalho citado, as crianças prematuras foram avaliadas após a alta da unidade neonatal ou mesmo do hospital, ao contrário da presente pesquisa. Assim, tal fato pode ter influenciado na menor frequência de atraso encontrada pelas referidas autoras, levantando a hipótese de uma possível influência da alta da unidade neonatal sobre o atraso do desenvolvimento.

Com relação à assistência pré-natal, a presente pesquisa evidenciou um percentual maior de crianças com desenvolvimento alterado entre aquelas cujas mães realizaram mais consultas prénatais. Uma possível justificativa para esse resultado seria o fato de que as mães que realizaram um maior número de consultas apresentaram mais complicações no período gestacional e, portanto, teriam adicionado fatores de risco ao desenvolvimento de seus filhos. De fato, $84,4 \%$ das mães que apresentaram hipertensão arterial e $64,7 \%$ das que desenvolveram infecções estavam entre as que realizaram mais consultas. No entanto, tais enfermidades não estiveram significativamente associadas ao desfecho de desenvolvimento.

Quanto às características biológicas do recémnascido, encontraram-se piores resultados de desenvolvimento conforme decrescia o peso de nascimento, estando esse resultado em concordância com estudos anteriores.1,16-19 O risco de repercussões graves para o desenvolvimento relaciona-se inversamente com a idade gestacional e o peso ao nascimento. 16,18,20 Segundo Aylward, 3 crianças prematuras extremas e/ou de extremo baixo peso ao nascer também estão sujeitas a alterações mais leves do comportamento motor, mesmo não apresentando lesões neurológicas graves. Em avaliações precoces, esses neonatos tendem a apresentar atraso no controle da cabeça e nas reações posturais, ${ }^{21}$ déficits da coordenação motora e distúrbios do tônus muscular. 5

Contrariamente ao relatado por outros autores, $1,2,10$ no presente estudo a idade gestacional não mostrou associação significante com o atraso do desenvolvimento motor. Entretanto, observou-se uma maior proporção de crianças com desenvolvimento alterado entre aquelas com idade gestacional igual ou menor que 32 semanas, as quais reconhecidamente compõem o grupo de maior risco para atraso do desenvolvimento. ${ }^{17} \mathrm{Na}$ presente pesquisa, a maior parte da amostra foi constituída por prematuros tardios, nascidos entre 35 e 36 semanas de idade gestacional.

Por outro lado, a idade pós-conceptual no momento da avaliação foi uma importante variável no presente estudo, onde crianças avaliadas em idades mais avançadas obtiveram maior frequência de alteração do desenvolvimento motor. Tal resultado justifica-se pelos pré-requisitos para a aplicação do TIMP, pois entre as crianças avaliadas mais tardiamente, estavam aquelas que demoraram mais a serem incluídas na pesquisa devido a sua gravidade clínica, tendo apresentado mais morbidades e permanecido mais tempo internadas, constituindo, 
assim, um grupo de maior risco para atraso do desenvolvimento motor.

Dentre as morbidades apresentadas pelas crianças, a hipóxia/asfixia perinatal e a displasia broncopulmonar apresentaram associação com o desenvolvimento motor alterado, confirmando resultados anteriores.1,2 A hipóxia/asfixia perinatal evolui, frequentemente, com leucomalácia periventricular, 3,20 que pode causar sequelas neuropsicomotoras importantes como paralisia cerebral.3,16,22 Por sua vez, a displasia broncopulmonar tem sido considerada como preditora de alterações motoras, ${ }^{23,24}$ pois sujeita as crianças a frequentes episódios de hipóxia, hipercapnia e acidose respiratória, podendo comprometer o sistema nervoso central. ${ }^{2}$

Quanto aos tratamentos realizados na unidade neonatal, a oxigenoterapia mostrou-se como um fator fortemente associado ao desfecho. A explicação pode ser a produção excessiva de radicais livres após o nascimento, a qual é intensificada com a oxigenoterapia. Segundo French et al., 25 o estresse oxidativo pode contribuir para a hipomielinização difusa encontrada na lesão da matriz branca periventricular, a qual está associada à lesão neurológica crônica em recém-nascidos prematuros e, consequentemente, o atraso do desenvolvimento motor.

No tecido pulmonar, os efeitos deletérios do oxigênio são potencializados pelo uso prolongado de ventilação mecânica, estando ambos relacionados com evolução para displasia broncopulmonar.12 A ventilação mecânica limita a variação de posição do neonato, 26 restringindo seus movimentos espontâneos, ${ }^{6}$ além de provocar alterações hemodinâmicas e na oxigenação tecidual, que podem favorecer lesões neurológicas, ${ }^{27}$ de modo que têm sido associada ao desenvolvimento motor em avaliações precoces $28 \mathrm{e}$ tardias. ${ }^{1,4}$

Embora o presente estudo tenha mostrado uma tendência a piores resultados motores com o aumento no tempo de oxigenoterapia e de permanência da criança na ventilação mecânica, não foi possível estabelecer uma relação causal direta desses fatores com atraso do desenvolvimento, pois crianças que utilizaram tais recursos por períodos prolongados foram também as mais graves. Entretanto, os resultados aqui encontrados concordam com os achados de Nicolau et al. ${ }^{28}$ que, avaliando prematuros na alta hospitalar através do TIMP, encontraram pior desempenho motor entre aqueles que permaneceram mais tempo em oxigenoterapia e suporte ventilatório.

Os tempos de permanência na Unidade Neonatal e na UTI foram avaliados com o intuito de investigar o impacto desses ambientes sobre o desenvolvimento motor. Ambas as variáveis estiveram associadas ao desfecho de desenvolvimento, havendo uma tendência evidente ao aumento do percentual de crianças com desenvolvimento alterado conforme aumentavam esses períodos, confirmando relatos anteriores. 1,18 Dados semelhantes estão presentes no estudo de Nicolau et al., ${ }^{28}$ que constatou que a internação prolongada compromete o desenvolvimento neuromotor em recém-nascidos pré-termo. Por outro lado, nos achados de Guimarães et al., 15 o tempo de internamento não mostrou associação significativa com o desenvolvimento motor atípico segundo o TIMP. No entanto, conforme sugerido pelos próprios autores, essa divergência poderia ser explicada pela utilização de diferentes critérios de análise na determinação das associações.

Um maior tempo de permanência na UTI neonatal expõe a criança a uma estimulação excessiva, provocando alterações no seu comportamento motor, 7,8 o qual pode ser influenciado, ainda, por posicionamento impróprio. 29 Ademais, procedimentos intrusivos e dolorosos levam a um comportamento aversivo, gerando torção do corpo e/ou flexão de braços e pernas, 30 contribuindo para alterações no padrão postural e na atividade espontânea dos membros.

O presente estudo apresentou como limitações metodológicas o viés de informação relacionado à subnotificação de alguns prontuários, a ausência de acompanhamento longitudinal e o tamanho reduzido da amostra, embora a mesma tenha correspondido à maior parte das crianças internadas no período de observação. Apesar dessas limitações, o ponto forte desta pesquisa foi seu caráter exploratório. A maioria dos estudos de avaliação do desenvolvimento motor de prematuros aborda crianças a partir de 12 meses de idade corrigida, deixando à margem o primeiro ano de vida que, devido à maior vulnerabilidade do sistema nervoso, é considerado crítico para o desenvolvimento, sendo também a fase ideal para instituição de medidas de prevenção e tratamento em virtude da maior plasticidade cerebral. ${ }^{9}$

Pesquisas de avaliação realizadas no período de internação são ainda mais escassas e este estudo procurou preencher essa lacuna, diferenciando-se de estudos anteriores ${ }^{15,28}$ que, embora tenham utilizado o mesmo instrumento para avaliar o desenvolvimento motor de prematuros, não o fizeram com uma amostra integralmente constituída por crianças internadas em unidade neonatal.

A partir dos achados da presente pesquisa, concluiu-se que, em prematuros de risco, as alterações do desenvolvimento motor podem se mani- 
festar precocemente, evidenciando-se como atrasos graves ou como desempenho motor limítrofe, o que justifica o início de estratégias de prevenção e estimulação ainda na unidade neonatal. Na amostra estudada, o peso ao nascer, a idade no momento da avaliação, a ocorrência de hipóxia e displasia broncopulmonar, além dos tempos de oxigenoterapia, ventilação mecânica e permanência na UTI neonatal associaram-se a alteração do desenvolvimento

\section{Referências}

1. Kreling KCA, Brito ASJ de, Matsuo T. Fatores perinatais associados ao desenvolvimento neuropsicomotor de recémnascidos de muito baixo peso. Pediatria (São Paulo). 2006; 28: 98-108.

2. Resegue R, Puccini RF, Silva EMK. Fatores de risco associados a alterações no desenvolvimento da criança. Pediatria (São Paulo). 2007; 29: 117-28.

3. Aylward GP. Neurodevelopmental outcomes of infants born prematurely. J Dev Behav Pediatr. 2005; 26: 427-40.

4. Kobaly K, Schluchter M, Minich N, Friedman H, Taylor HG, Wilson-Costello D, Hack M. Outcomes of extremely low birth weight $(<1 \mathrm{~kg})$ and extremely low gestational age ( $<28$ weeks) infants with bronchopulmonary dysplasia: effects of practice changes in 2000 to 2003 . Pediatrics. 2008; 121: 73-81.

5. Brown NC, Doyle LW, Bear MJ, Inder TE. Alterations in neurobehavior at term reflect differing perinatal exposures in very preterm infants. Pediatrics. 2006; 118: 2461-71.

6. Moster D, Lie RT, Markestad T. Long-term medical and social consequences of preterm birth. N Engl J Med. 2008; 359: $262-73$.

7. Blackburn S. Environmental impact of the NICU on developmental outcomes. J Pediatr Nurs. 1998; 13: 279-89.

8. Vandenberg K. Individualized developmental care for high risk newborns in the NICU: A practice guideline. Early Hum Dev. 2007; 83: 433-42.

9. Als H, Duffy FH, McAnulty GB, Rivkin MJ, Vajapeyam S, Mulkern RV, Warfield SK, Huppi PS, Butler SC, Conneman $\mathrm{N}$, Fischer C, Eichenwald EC. Early experience alters brain function and structure. Pediatrics. 2004; 113: 846-57.

10. Mercuri E, Ricci D, Romeo DM. Neurological and visual assessments in very and late low-risk preterm infants. Early Hum Dev. 2012; 88: S31-S33.

11. Spittle AJ, Doyle LW, Boyd RN. A systematic review of the clinimetric properties of neuromotor assessments for preterm infants during the first year of life. Dev Med Child Neurol. 2008; 50: 254-66.

12. Jobe AH, Bancalari E. Bronchopulmonary dysplasia. Am J Respir Crit Care Med. 2001; 163: 1723-9.

13. Campbell SK. The test of infant motor performance: test user's manual version 2.0. Chicago, IL: Infant Motor Performance Scales; 2005.

14. Flegel J, Kolobe THA. Predictive validity of the Test of Infant Motor Performance as measured by the BruininksOseretsky Test of Motor Proficiency at school age. Phys Ther. 2002; 82: 762-71. motor. Entretanto, estudos longitudinais, com amostras maiores e análise multivariada, são recomendados para verificar a persistência dos distúrbios motores e confirmar o valor prognóstico das avaliações precoces, possibilitando, através da eliminação das variáveis de confusão, a identificação dos fatores verdadeiramente implicados no atraso do desenvolvimento motor.

15. Guimarães CLN, Reinaux CM, Botelho ACG, Lima GMS, Cabral Filho JE. Desenvolvimento motor avaliado pelo Test of Infant Motor Performance: comparação entre lactentes pré-termo e a termo. Rev Bras Fisioter. 2011; 15: 357-62.

16. Mercier CE, Dunn MS, Ferrelli KR, Howard DB, Soll RF and the Vermont Oxford Network ELBW Infant Follow-Up Study Group. Neurodevelopmental outcome of extremely low birth weight infants from the Vermont Oxford Network: 1998-2003. Neonatology. 2010; 97: 329-38.

17. Vohr BR, Wright LL, Poole WK, McDonald SA. Neurodevelopmental outcomes of extremely low birth weight infants <32 weeks' gestation between 1993 and 1998. Pediatrics. 2005; 116: 635-43.

18. Ruiz-Extremera A, Robles-Vizcaino C, Salvatierra-Cuenca MT, Ocete E, Lainez C, Benitez A, Cruz F, Miranda MT, Salmerón J. Neurodevelopment of neonates in neonatal intensive care units and growth of surviving infants at age 2 years. Early Hum Dev. 2001; 65 (Suppl.): S119-S32.

19. Lin YC, Lin YJ, Lin CH. Growth and neurodevelopmental outcomes of extremely low birth weight infants: a single center's experience. Pediatr Neonatol. 2011; 52: 342-8.

20. Silveira RC, Procianoy RS. Lesões isquêmicas cerebrais no recém-nascido pré-termo de muito baixo peso. J Pediatr (Rio J). 2005; 81 (Supl. 1): S23-S32.

21. Cameron EC, Maehle V. Comparison of active motor items in infants born preterm and infants born full term. Pediatr Phys Ther. 2006; 18: 197-203.

22. Resch B, Neubauer K, Hofer N, Resch E, Maurer U, Haas J, Müller W. Episodes of hypocarbia and early-onset sepsis are risk factors for cystic periventricular leukomalacia in the preterm infant. Early Hum Dev. 2012; 88: 27-31.

23. Shepherd EG, Knupp AM, Welty SE, Susey KM, Gardner WP, Gest AL. An interdisciplinary bronchopulmonary dysplasia program is associated with improved neurodevelopmental outcomes and fewer rehospitalizations. J Perinatol. 2012; 32: 33-8.

24. Natarajan G, Pappas A, Shankaran S, Kendrick DE, Das A, Higgins RD, Laptook AR, Bell EF, Stoll BJ, Newman N, Hale EC, Bara R, Walsh MC. Outcomes of extremely low birth weight infants with bronchopulmonary dysplasia: Impact of the physiologic definition. Early Hum Dev. 2012; 88: 509-15.

25. French HM, Reid M, Mamontov P, Simmons RA, Grinspan JB. Oxidative stress disrupts oligodendrocyte maturation. J Neurosci Res. 2009; 87: 3076-87. 
26. Samsom JF, Groot L, Bezemer D, Lafeber HN, Fetter WPF Muscle power development during the first year of life predicts neuromotor behavior at 7 years in preterm born high-risk infants. Early Hum Dev. 2002; 68: 103-18.

27. Limperopoulos C, Gauvreau KK, O'Leary H, Moore M, Bassan H, Eichenwald EC, Soul JS, Ringer SA, Di Salvo DN, Plessis AJ. Cerebral hemodynamic changes during intensive care of preterm infants. Pediatrics. 2008; 122: 1006-3.

28. Nicolau CM, Costa APB M, Hazime HO, Krebs VLJ. Desempenho motor em recém-nascidos pré-termo de alto risco. Rev Bras Crescimento Desenv Hum. 2011; 21: 327 34.

Recebido em 19 de outubro de 2012

Versão final apresentada em 25 de fevereiro de 2013

Aprovado em 27 de abril de 2013
29. Sweeney KJ, Gutierrez T. Musculoskeletal implications of preterm infant positioning in the NICU. J Neonatal Nurs. 2002; 16: 58-70.

30. Sehgal A, Stack J. Developmentally supportive care and NIDCAP. Indian J Pediatr. 2006; 73: 1008-10. 